\title{
Apoptosis induced by disruption of the actin cytoskeleton is mediated via activation of CD95 (Fas/APO-1)
}

\author{
D Kulms ${ }^{1}$, H Düßmann ${ }^{2}$, B Pöppelmann ${ }^{1}$, S Ständer ${ }^{1}$, \\ A Schwarz ${ }^{1}$ and T Schwarz ${ }^{*, 1}$ \\ 1 Ludwig Boltzmann Institute for Cell Biology and Immunobiology of the Skin, \\ Department of Dermatology, University Münster, D-48149 Münster, Germany \\ 2 Interdisciplinary Center for Clinical Research, University Münster, D-48149 \\ Münster, Germany \\ * Corresponding author: Thomas Schwarz, Department of Dermatology, \\ University Münster, Von-Esmarchstrasse 58, D-48149 Münster, Germany. \\ Tel: +49-251-8356565; Fax: +49-251-8358579; \\ E-mail: schwtho@uni-muenster.de
}

Received 27.8.01; revised 26.11.01; accepted 17.12.01

Edited by PH Krammer

\begin{abstract}
Activation of the death receptor CD95 by its ligand or by UV radiation is associated with receptor clustering. The mechanism underlying this clustering is mostly unclear. Here we show that although disruption of the actin cytoskeleton by cytochalasin B (СyB) itself induces moderate apoptosis, it enhances apoptosis in HeLa cells induced either by UV radiation or an agonistic anti-CD95 antibody. CyB augments UV-induced apoptosis independently of UV-mediated DNA damage, since induction of DNA repair by exogenous DNA repair enzymes did not alter its enhancing effect. Inhibition of caspase-8, the most upstream caspase in CD95 signaling, blocked the apoptotic effect of CyB and the enhancing effect on UV-and CD95-induced apoptosis. Confocal laser scanning microscopy revealed that (i) CyB induces $\mathrm{CD} 95$ clustering, (ii) enhances UV-induced CD95 clustering, and (iii) CD95 clusters colocalize with disrupted actin filaments, suggesting a link between receptor clustering and actin rearrangement. Disruption of CD95 signaling by a dominant negative mutant of the signaling protein FADD protected from CyB-induced apoptosis and prevented the UV-enhancing effect. Accordingly, both the apoptotic and the enhancing effect of $\mathrm{CyB}$ was reduced in epidermal cells obtained from CD95 deficient mice (Ipr) when compared to wild-type mice. These data suggest that disruption of the cytoskeleton causes apoptosis via activation of CD95 and enhances UV-induced apoptosis, possibly via aiding receptor clustering.

Cell Death and Differentiation (2002) 9, 598-608. DOI: 10.1038/ sj/cdd/4401002
\end{abstract}

Keywords: UV radiation; death receptor; cytoskeleton; apoptosis; CD95/Fas

Abbreviations: CyB, cytochalasin B; Ab, antibody; FITC, fluorescein-isothiocyanate; FLIP, FLICE inhibitory protein; TRITC, tetramethylrhodamine-isothiocyanate

\section{Introduction}

Apoptosis is a cell death process which can be induced by several stimuli, such as growth factor deprivation, ionizing radiation, UV radiation, other DNA-damaging agents, or by triggering specific cell surface receptors, the so-called death receptors. Death receptors belong to the tumor necrosis factor receptor gene superfamily, which is characterized by similar cysteine-rich extracellular domains. ${ }^{1}$ Among the many death receptors described, CD95 (Fas/APO-1) appears to be the most important one. Engagement of CD95 by agonistic antiCD95 antibodies or by the cognate ligand, CD95L, induces apoptosis. ${ }^{1}$ The main death pathway initiated by activation of CD95 involves a series of death-associated molecules, including the Fas-associated death domain-containing protein (FADD), an adaptor protein that is recruited to the CD95 receptor upon its engagement. ${ }^{2,3}$ FADD then binds to and activates procaspase- $8,{ }^{4,5}$ which is the initial step of a proteolytic cascade triggering the activation of other downstream caspases such as caspases-3, $-6,-7$ and $-9 .^{6}$

In contrast to CD95-induced cell death, apoptosis induced by UV radiation is a much more complex process, in which several different pathways are involved. ${ }^{7}$ A major determinant whether a cell undergoes apoptosis following UV exposure is the severity of DNA damage induced by UV radiation. This conception is supported by the observation that the reduction of DNA damage following DNA repair is associated with a decrease of apoptotic cell death. ${ }^{8-11}$ Furthermore, the tumor suppressor gene $p 53$, which is activated by DNA damage, is critically involved in UV-induced apoptosis, since mice lacking functional p53 reveal significantly fewer apoptotic keratinocytes in the epidermis following UV exposure in comparison to wild-type mice. ${ }^{12}$ On the other hand, UV radiation was found to exhibit the capacity to directly activate CD95 without need of the ligand. ${ }^{13,14}$ This appears to be functionally relevant, since inhibition of caspase-8, the crucial upstream caspase in CD95 signaling, as well as overexpression of a dominant negative mutant for FADD was associated with a reduction of UV-induced apoptosis. ${ }^{13}$ However, the mechanism by which UV radiation directly activates the CD95 receptor still remains to be determined.

When triggering CD95, UV radiation induces clustering of this death receptor ${ }^{13,14}$ which is also observed when CD95 is activated by CD95L or by agonistic antibodies. ${ }^{13}$ Induction of receptor clustering by UV radiation is not specific for CD95, since Rosette and Karin initially demonstrated this phenomenon for other receptors including those for epidermal growth factor, interleukin-1 and tumor necrosis factor $\alpha$, another death ligand related to CD95L. ${ }^{15}$ Based on this observation, Rosette and Karin predicted that any receptor whose activation mechanism involves multimerization should be activatable by UV radiation. Induction of CD95 clustering by UV radiation appears to be functionally relevant since inhibition of receptor clustering, which can be achieved by 
keeping cells at low temperature $\left(4-10^{\circ} \mathrm{C}\right)$ during UV exposure, is associated with a reduction of apoptotic cell death. ${ }^{13,14,16}$ However, the mechanism by which UV radiation or the respective ligands induce receptor clustering still remains largely unclear.

Receptor clustering resembles a kind of polarizing phenomenon requiring asymmetric organization of the plasma membrane. ${ }^{17}$ The capacity of a cell to polarize is directly linked to an interaction between the membrane and the cytoskeleton. ${ }^{18}$ Accordingly, interactions between plasma membranes and the cytoskeleton play a crucial role in various cell functions ${ }^{19-21}$ and this may also apply for death receptor mediated apoptosis. ${ }^{17}$ There is no doubt that apoptosis has dramatic effects on the cytoskeleton. Through cleavage of intermediate filament proteins, apoptosis alters the integrity of the cytoskeleton and thereby affects the overall cellular structure..$^{22-24}$ On the other hand, disruption of the cytoskeleton by itself can induce or accelerate apoptosis. ${ }^{25-27}$ Although the detailed underlying mechanisms still remain to be determined, these observations challenged the current model that regards cytoskeletal alterations only as a consequence and not as a cause of apoptosis, suggesting that the cytoskeleton may not only be a target of the suicide mechanisms of apoptosis but also an initiator of programmed cell death. ${ }^{26}$

In consideration of these findings, we were interested in studying the impact of the perturbation of the cytoskeleton on CD95 receptor clustering and on UV-induced apoptosis. Here we show that disruption of the actin cytoskeleton by cytochalasin B (CyB) itself induces moderate apoptosis, but considerably enhances both UV- and anti-CD95 antibody driven apoptosis in the epithelial cell line HeLa. Treatment of HeLa cells with CyB induces clustering of CD95. CD95 clusters colocalized with disrupted actin filaments. CD95 clustering mediated by CyB appears to be functionally relevant since the apoptotic effect induced by CyB was inhibited in cells overexpressing a dominant negative mutant of FADD. Taken together, these data suggest that disruption of the cytoskeleton causes apoptosis via activation of CD95, demonstrating that death receptors are not only triggered by external stimuli but also by signals originating from within the cell. Furthermore, cytoskeletal disruption enhances UV-induced apoptosis possibly by aiding death receptor clustering.

\section{Results}

\section{Incubation of HeLa cells with cytochalasin B induces apoptosis and enhances UV- and CD95-induced apoptosis}

Exposure of HeLa cells to $50 \mu \mathrm{g} / \mathrm{ml}$ CyB moderately induced apoptosis as determined by a cell death detection ELISA (Figure 1). Exposure of HeLa cells to $400 \mathrm{~J} / \mathrm{m}^{2} \mathrm{UV}$ radiation or $1 \mu \mathrm{g} / \mathrm{ml}$ of an agonistic anti-CD95 antibody caused apoptosis to a much higher extent. Pre-exposure of HeLa cells to $50 \mu \mathrm{g} /$ $\mathrm{ml}$ CyB for $1 \mathrm{~h}$ enhanced both UV- and CD95-induced apoptosis. Similar observations were obtained when annexin $V$ staining was used as a read-out system for apoptosis (data not shown).

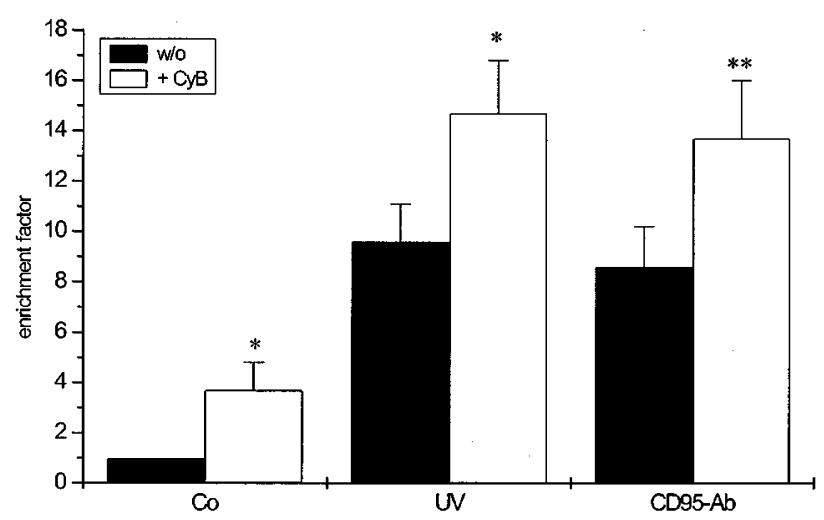

Figure 1 Cytochalasin B induces apoptosis and enhances UV- and CD95mediated cell death. HeLa cells were left untreated $\left(C_{0}\right)$ or preincubated for $1 \mathrm{~h}$ with CyB $(50 \mu \mathrm{g} / \mathrm{ml})$, followed by UV irradiation (UV) or stimulation with an agonistic anti-CD95 antibody (CD95-Ab). After incubation at $37^{\circ} \mathrm{C}$ for $16 \mathrm{~h}$, apoptosis was evaluated using a cell death detection ELISA. Apoptosis is reflected by the enrichment of nucleosomes in the cytoplasm, shown on the $y$ axis (mean \pm S.D. of seven independently performed experiments). ${ }^{*} P<0.001$ vs w/o; ${ }^{* *} P<0.005$ vs w/o

As recently shown, UV-induced apoptosis is a much more complex process than CD95-mediated apoptosis, since several pathways are involved in UV-driven cell death. ${ }^{7} \mathrm{UV}$ induced nuclear DNA damage and direct activation of death receptors, such as CD95, by UV radiation contribute independently to UV-mediated apoptosis. ${ }^{8}$ Since CyB enhanced UV-induced cell death, we were interested in clarifying whether CyB enhances the membrane or the nuclear driven pathway of UV-mediated apoptosis. To address this issue, we utilized a system which enables rapid removal of DNA damage by induction of DNA repair via addition of the exogenous DNA repair enzyme photolyase encapsulated into liposomes which allow penetration into cells both in vitro and in vivo. ${ }^{28}$ Photolyase binds to an UV-induced cyclobutane thymine dimer in DNA and catalyzes its splitting by electron transfer from absorbing wavelengths above $320 \mathrm{~nm}$ (photoreactivating light), a process called photoreactivation. ${ }^{29}$

To determine first whether photoreactivation effectively reduces DNA damage, Southwestern dot-blot analysis using an antibody directed against cyclobutane thymine dimers was performed. No dimers were present in genomic DNA of untreated cells (Figure 2B, dot 1). In contrast, dimers were detected in HeLa cells exposed to UV radiation (dot 2) but were significantly reduced when UV-irradiated cells were treated with the combination of photolyase plus photoreactivating light (dot 3 ). The addition of empty liposomes had no effect on the amount UV-induced DNA lesions (dot 4). Thus, these data indicate that photoreactivation effectively enhances DNA repair by removing cyclobutane thymine dimers in UV-exposed HeLa cells.

To determine the impact of photoreactivation on the apoptosis enhancing effect of CyB, HeLa cells were exposed to UV radiation in the absence or presence of CyB. Immediately after UV exposure, photoreactivation was induced. In accordance with our previous findings, ${ }^{8}$ photoreactivation reduced UV-induced apoptosis by around 
A

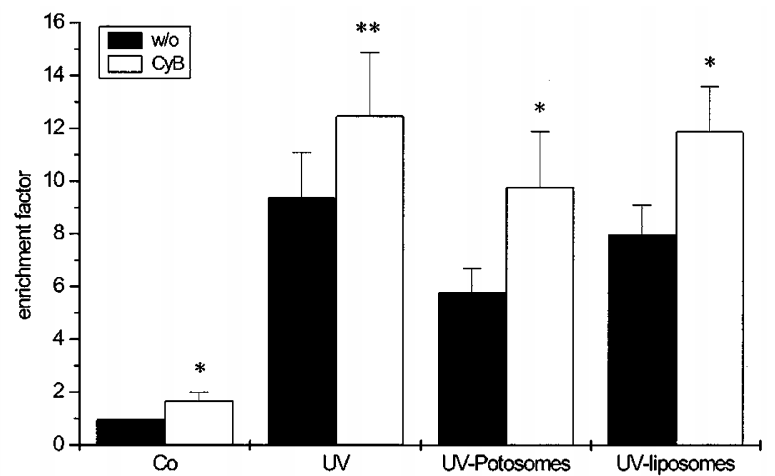

B

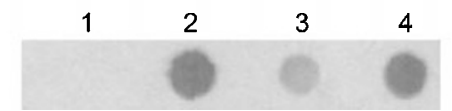

Figure 2 The effect of CyB on UV-induced apoptosis is not affected by enhanced removal of DNA-damage. HeLa cells were preincubated in the absence or presence of CyB for $1 \mathrm{~h}$ and subsequently exposed to UV irradiation (UV). Immediately thereafter, Photosomes or empty liposomes were added and cells kept for $1 \mathrm{~h}$ at $37^{\circ} \mathrm{C}$ in the dark. Finally, photoreactivation was carried out by irradiating cells with photoreactivating light. (A) Apoptosis was measured $16 \mathrm{~h}$ later with a cell death detection ELISA. The enrichment factor was used as a parameter of apoptosis and is given as the mean \pm S.D. of six independently performed experiments. ${ }^{*} P<0.001 \mathrm{vs} \mathrm{w} / 0$; ${ }^{* *} P<0.005 \mathrm{vs} \mathrm{w} / 0$. (B) $1 \mathrm{~h}$ after photoreactivation genomic DNA was extracted and Southwestern dot-blot analysis was performed using an antibody directed against thymine dimers. Dot 1: untreated cells; dot 2: UV-irradiated cells; dot 3: UV-exposed cells treated with Photosomes; dot 4: UV-exposed cells treated with empty liposomes

$50 \%$, while empty liposomes had no effect (Figure $2 A$ ). In addition, the enhancing effect of CyB on UV-induced apoptosis remained the same after photoreactivation. Since removal of cyclobutane thymine dimers did not alter the enhancing effect of CyB, this indicates that CyB augments UV-induced apoptosis independently of DNA damage.

\section{The effect of cytochalasin B on apoptosis is blocked by inhibition of caspase-8}

To further characterize the enhancing effect of CyB on UVand CD95-induced apoptosis, we analyzed the involvement of caspases by using caspase inhibitors. As shown in Figure 3, pretreatment of HeLa cells with the broad-spectrum caspase inhibitor zVAD completely blocked apoptosis induced by CyB (Figure 3A), by UV (Figure 3B) or by an anti-CD95 antibody (Figure $3 C$ ). Likewise, the enhancing effect of CyB on UV-and CD95-induced apoptosis was inhibited in the presence of zVAD, indicating the crucial involvement of caspases. The caspase-8 inhibitor zIETD completely suppressed CD95mediated apoptosis, while UV-induced cell death was only partially inhibited, confirming previous observations. ${ }^{8}$ As caspase- 8 is critically involved in CD95 signaling, activation of caspase-8 during UV-induced apoptosis appears to be most likely due to the direct triggering of CD95 by UV radiation, ${ }^{13,14}$ while the remaining apoptotic activity is most likely mediated via UV-induced DNA damage in a caspase-8 independent way. ${ }^{8}$ zIETD completely blocked the apoptotic effect of CyB as well as the augmenting effect of CyB on
A
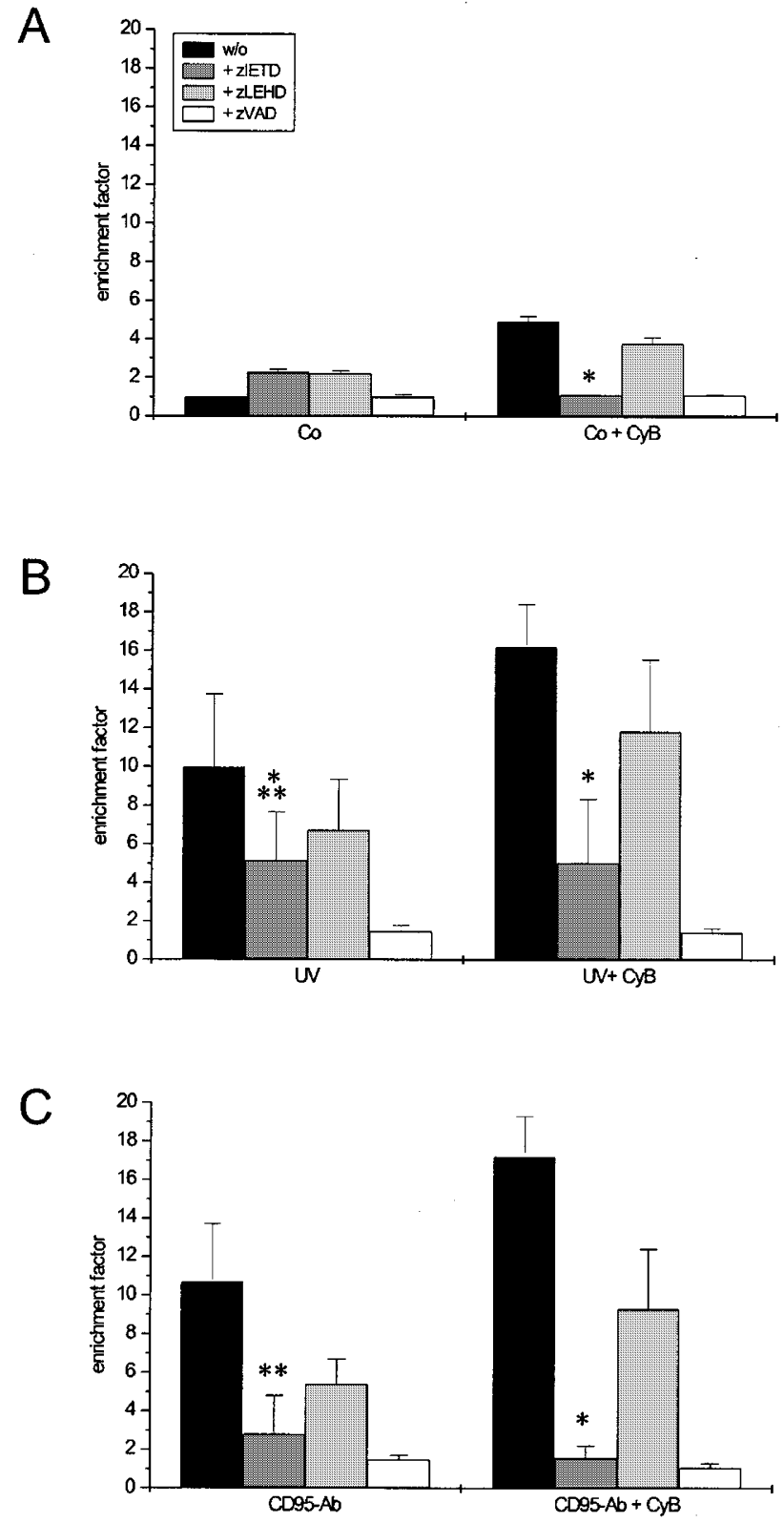

Figure 3 Caspase inhibitors differentially affect the effect of CyB. Cells were treated with one of the caspase inhibitors (zVAD, zLEHD, zIETD; $20 \mu \mathrm{M}$ each), followed by exposure to CyB for $1 \mathrm{~h}$. Finally, cells were exposed to UV radiation or treated with an agonistic anti-CD95 antibody (CD95-Ab). Control cells were left untreated. After $16 \mathrm{~h}$ apoptosis was evaluated with a cell death detection ELISA. The enrichment factor was used as a parameter of apoptosis and is given as the mean \pm S.D. of three independently performed experiments. ${ }^{*} P<0.001$ vs $\mathrm{w} / 0$; ${ }^{* *} P<0.005$ vs $\mathrm{w} / 0 ;{ }^{* *} P<0.05$ vs $\mathrm{w} / \mathrm{o}$

CD95- and UV-induced apoptosis. It is important to note that in all three cases, CyB-, UV- and CD95-induced apoptosis, death rates were reduced to the same degree, irrespective of whether the apoptosis rate was enhanced by preincubation with CyB or not. This demonstrates that caspase-8, the most upstream caspase in CD95 triggered apoptosis, is crucially involved in the enhancing effect of CyB on apoptosis. It further indicates that CyB indeed enhances CD95- and UV-induced apoptosis rather than exerting just an additive effect. 
In contrast, inhibition of the downstream caspase- 9 by the specific tetrapeptide zLEHD had almost no effect on CyB-induced apoptosis and only slightly reduced the enhancing effect of CyB on either UV- or CD95-induced apoptosis (Figure 3). Although a moderate reduction of the apoptosis rate could be observed, the degree of increased apoptosis caused by CyB remained the same, indicating that CyB triggers or enhances pathways located in the cytoplasm close to the membrane and upstream within the apoptotic machinery.

\section{Cytochalasin B induces and enhances the cleavage of caspase-3 and Bid and the release of cytochrome $c$}

The data so far indicate that caspase- 8 is essentially involved in the apoptosis enhancing effect of CyB. Activated caspase-8 can activate downstream caspases, including caspase-3, either by direct cleavage or indirectly by cleaving Bid and inducing cytochrome $c$ release from the mitochondria. ${ }^{30}$ To analyze the effect of CyB on cleavage of caspase-3 and Bid and on cytochrome $c$ release, HeLa cells were either stimulated with $\mathrm{CyB}$ alone or prestimulated with CyB followed by exposure to UV radiation or anti-CD95 antibody. Cytoplasmic and mitochondrial protein extracts were obtained and subjected to Western blot analysis as shown in Figure 4. Exposure of cells to CyB alone results in moderate cleavage of either caspase-3 or Bid and a weak release of cytochrome $c$ from mitochondria into the cytoplasm. Induction of apoptosis by UV radiation or activation of CD95 by an agonistic antibody resulted in a much more pronounced cleavage of caspase-3 and Bid and accordingly caused stronger cytochrome $c$ release. Both UV- and CD95-induced activation of the proapoptotic Bid protein or caspase-3 were clearly enhanced upon pretreatment with CyB. The same was observed for cytochrome $c$ release, since the cytochrome $c$ levels were found enhanced in the cytoplasmic but correspondingly reduced in the mitochondrial extracts (Figure 4). The antibodies used for the detection of either Bid or caspase-3 only recognize the inactive proforms of the respective proteins, so that in both cases cleavage is reflected by fading

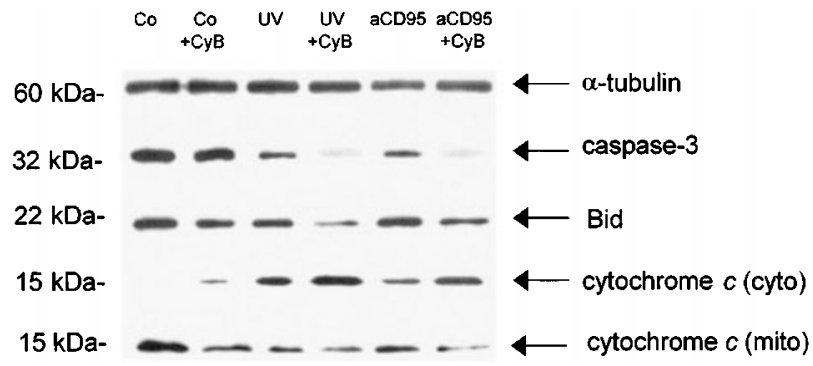

Figure 4 CyB causes cleavage of caspase-3 and followed by release of cytochrome $c$. Cells were left untreated, incubated with CyB, UV-irradiated in the absence or presence of CyB for $1 \mathrm{~h}$, or treated with an agonistic anti-CD95 antibody in the absence or presence of CyB for $1 \mathrm{~h}$. After $16 \mathrm{~h}$, cytoplasmic protein extracts were subjected to Western blot analysis using antibodies directed against caspase-3, Bid, or cytochrome $c$. In addition, mitochondrial protein extracts were obtained and blotted with an antibody directed against cytochrome $c$. Equal loading was monitored with an antibody directed against $\alpha$-tubulin of the protein band. Decrease of the respective protein bands was not due to differences in protein loading since equal protein levels were detected upon reprobing the membrane with an antibody directed against $\alpha$-tubulin. Taken together, the data indicate that caspase- 8 dependent cleavage of Bid and caspase- 3 is enhanced by $\mathrm{CyB}$, thereby augmenting the mitochondrial and the cytoplasmic apoptosis pathways triggered by UV and activation of CD95.

\section{Cytochalasin B does not modulate FLIP expression}

It has been demonstrated that CD95-mediated apoptosis can be modulated at the level of caspase- 8 by c-FLIP (FLICE inhibitory protein) ${ }^{31}$ which is an endogenous inhibitor of caspase- $8 .^{32}$ Since the balance between the expression levels of c-FLIP and caspase- 8 may determine the sensitivity of a cell to a CD95-mediated killing, we studied whether CyB enhances apoptosis by downregulating FLIP expression. HeLa cells were either stimulated with CyB alone or prestimulated with CyB followed by exposure to UV radiation or anti-CD95 antibody. Cytoplasmic protein extracts were subjected to Western blot analysis using an antibody directed against c-FLIP (Figure 5). CyB neither alone nor in combination with UV radiation or the anti-CD95 antibody was able to downregulate FLIP expression at significant levels.

\section{Cytochalasin B induces clustering of CD95 and enhances clustering induced by UV radiation and anti-CD95 antibodies}

A critical event during the activation of CD95 either by UV radiation, by its ligand or agonistic antibodies is the induction of receptor clustering as demonstrated by confocal laser scanning microscopy. ${ }^{13,14}$ Although the mechanism underlying receptor clustering is poorly understood, ${ }^{15}$ CD95 clustering appears to be functionally relevant since inhibition of clustering by exposing cells to UV radiation at low temperature $\left(4-10^{\circ} \mathrm{C}\right)$ attenuates this UV response..$^{8,13,16}$ Since, as demonstrated in Figure 3, apoptosis induced by CyB is critically dependent on caspase-8, the proximal caspase in the death-inducing signaling complex of CD95, we were interested in studying whether disruption of the cytoskeleton by CyB correlates with clustering of CD95 and/or enhances CD95 clustering induced by UV or anti-CD95antibody treatment.

To address this issue, HeLa cells were exposed to CyB alone, to UV radiation or to the agonistic anti-CD95

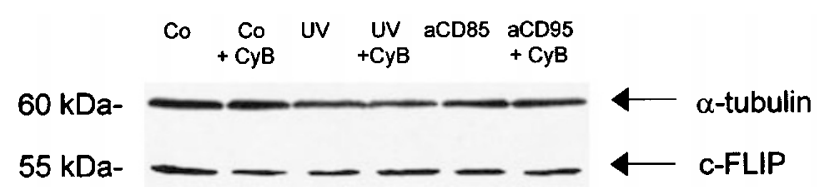

Figure 5 CyB does not modulate FLIP expression. Cells were left untreated, incubated with CyB, UV-irradiated in the absence or presence of CyB for $1 \mathrm{~h}$, or treated with an agonistic anti-CD95 antibody in the absence or presence of CyB for $1 \mathrm{~h}$. After $16 \mathrm{~h}$, cytoplasmic protein extracts were subjected to Western blot analysis using an antibody directed against FLIP. Equal loading was monitored with an antibody directed against $\alpha$-tubulin 
antibody in the absence or presence of CyB. Two hours later cells were double-stained with a FITC-conjugated antibody directed against CD95 and with TRITC-conjugated phalloidin and analyzed by confocal laser scanning microscopy. As shown in Figure 6, unstimulated cells (Co) revealed a filamentous actin skeleton and a diffuse

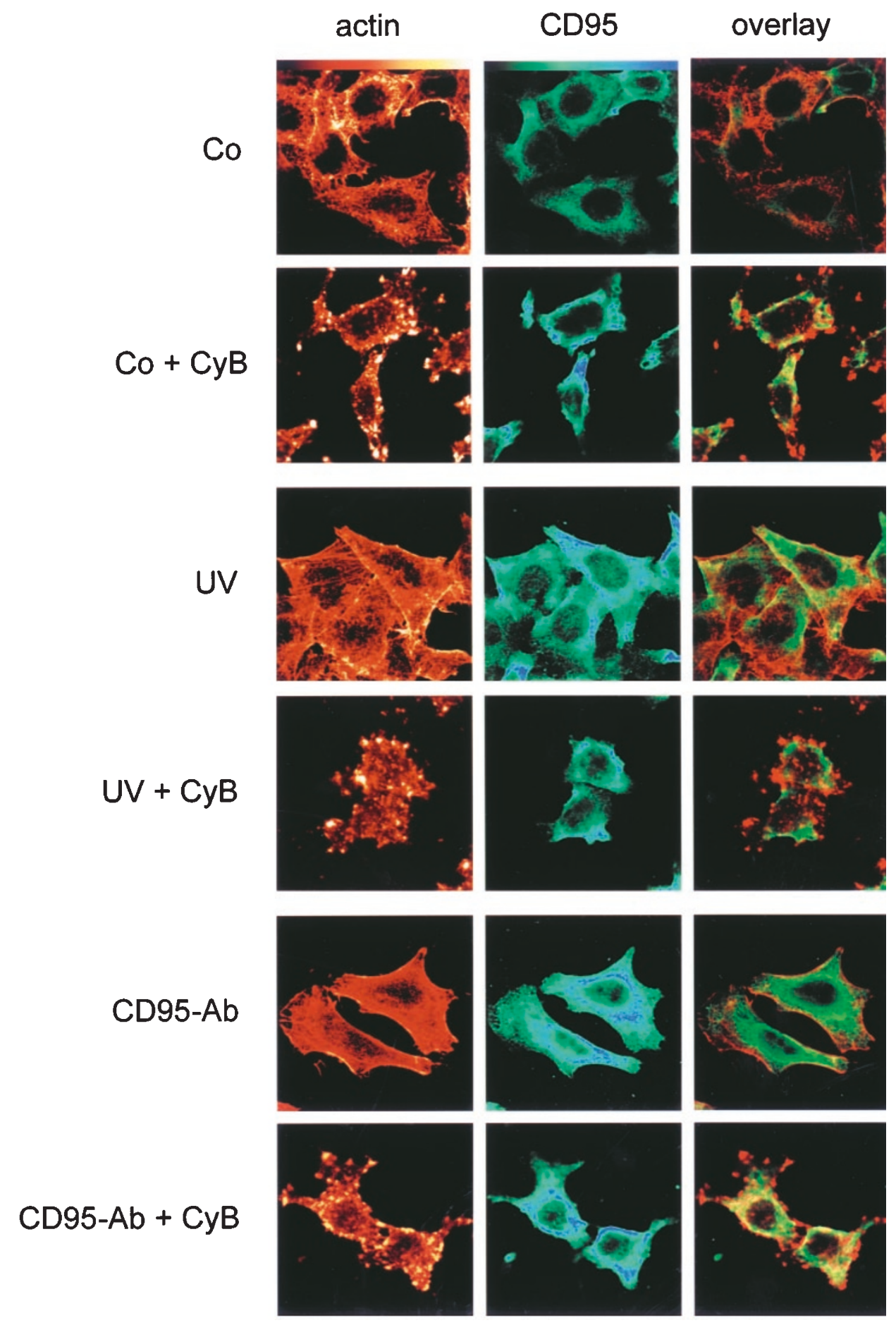

Figure 6 CyB causes disruption of the filamentous actin cytoskeleton and induces clustering of CD95. HeLa cells were left untreated or stimulated with CyB for $1 \mathrm{~h}$. Thereafter, cells were either left untreated or exposed to UV radiation $\left(400 \mathrm{~J} / \mathrm{m}^{2}\right)$ or to an agonistic anti-CD95 antibody. CD95 receptor staining was performed $2 \mathrm{~h}$ later using an antibody directed against CD95 (murine anti-human IgG) followed by incubation with an FITC-conjugated secondary rat-anti-mouse lgG (left column). Subsequently, actin was stained with TRITC-labeled phalloidin (mid column). Fluorescence was detected by confocal laser scanning microscopy. The images shown represent an object area of $64 \times 64 \mu \mathrm{m}^{2}$. The fluorescence intensity is indicated by the respective pseudocolor scale bars on top of the controls. The overlays of FITC (green) and TRITC (red) fluorescence are shown in the right column 
staining of CD95. Exposure to CyB alone (Co+CyB) resulted in disruption of the filamentous actin structures and assembly of depolymerized actin in the peripheral regions of the cytoplasm close to the cell membrane. At the same time, clustering of CD95 was observed. Overlay analysis localized the CD95 clusters to the same spots where actin aggregation was formed at the cell membrane. This suggests that aggregation of nonfilamentous actin after disruption by CyB coincides with clustering of the cell surface death receptor CD95, which thereby might trigger the intracellular apoptotic signal.

Stimulation of cells with UV radiation or the anti-CD95 antibody alone resulted in a moderate rearrangement of the intracellular actin skeleton after $2 \mathrm{~h}$. The highly polymerized actin filaments of the control cells exhibited a more diffuse appearance. Furthermore, slight actin aggregation at the cell periphery could be observed. At the same time points, CD95 clustering was detected after UV irradiation as well as after stimulation with the anti-CD95-antibody. This suggests that translocation of intracellular actin might be a prerequisite for the clustering of the cell surface bound death receptor CD95, thereby inducing the apoptotic pathway. While UV-induced clustering of CD95 seems to be rather random, evidenced by a more spotty distribution of CD95 clusters, activation of CD95 by the agonistic antibody seems to follow a more organized pattern, as indicated by a circular arrangement of CD95 clusters on the cell surface. In both cases of UV- or anti-CD95 antibodyinduced apoptosis, pretreatment with $\mathrm{CyB}$ enhanced death receptor aggregation (UV+CyB, CD95-Ab+CyB). Again, CD95 clusters colocalized mostly with disrupted actin filaments, as shown in overlay analysis. Taken together, these observations support the hypothesis that CyB may (i) induce CD95 clustering, (ii) facilitate CD95 clustering induced either by UV radiation or by the agonistic antiCD95-antibody and (iii) that receptor clustering may be closely connected to actin rearrangement.

\section{Transfection of cells with a FADD-dominant negative mutant prevents apoptosis induced by CyB}

If, as suggested by the data presented above, CyB induces and enhances apoptosis by triggering the CD95 receptor, the effect should be prevented, or at least reduced, if the CD95 signaling pathway was to be disrupted. Therefore, HeLa cells were transiently transfected with the FADD dominant negative mutant construct pcDNA3-FADD-DN or with the empty vector pcDNA3. Cells were exposed to UV radiation or to the anti-CD95 antibody either in the absence or presence of CyB $24 \mathrm{~h}$ after transfection, and apoptosis was measured using the cell death detection ELISA (Figure 7A). Transient transfection with the FADD-DN mutant construct protected HeLa cells from CyB-induced apoptosis. Both UV- and antiCD95 antibody-induced apoptosis were also significantly reduced in FADD-DN transfected cells. Accordingly, the enhancing effect of CyB on UV- and CD95-induced cell death was lost upon transfection with the FADD-DN construct, underlining the involvement of CD95 activation in CyB enhanced apoptosis.

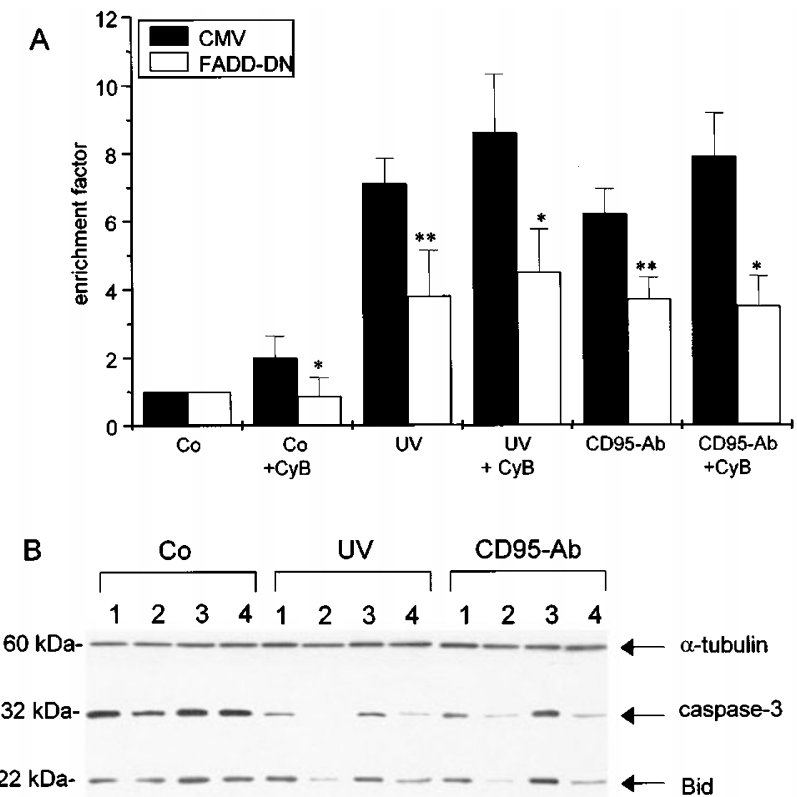

Figure 7 The enhanced apoptotic effect caused by CyB alone or in combination with either UV irradiation or CD95 activation is markedly reduced in cells transiently transfected with a FADD dominant negative mutant. Cells were transiently transfected with either the empty pcDNA3-CMV vector or pcDNA3-FADD-DN construct. (A) Cells of each transfection were left unstimulated or prestimulated with CyB. After $1 \mathrm{~h}$, cells were either left untreated for control purposes or treated with either $400 \mathrm{~J} / \mathrm{m}^{2} \mathrm{UV}$ or with an agonistic antibody against CD95. Apoptotic cell death was evaluated $16 \mathrm{~h}$ later using a cell death detection ELISA. The enrichment factor was used as a parameter of apoptosis and is given as the mean \pm S.D. of three independently performed experiments. (B) Cytoplasmic protein extracts from cells transfected either with the empty pcDNA3-CMV vector (lanes 1 and 2) or the pcDNA3-FADD-DN construct (lanes 3 and 4) were analyzed on $12 \%$ SDS-PAGE. Samples run in lanes 2 and 4 were incubated with CyB for $1 \mathrm{~h}$ prior to the above indicated stimulation for $16 \mathrm{~h}$. Western blot analysis was performed by reprobing the same membrane with antibodies against caspase3 or Bid. Equal loading was monitored with an antibody directed against $\alpha$ tubulin. ${ }^{*} P<0.001$ vs $\mathrm{CMV} ;{ }^{* *} P<0.01$ vs $\mathrm{CMV}$

The DNA fragmentation data were also confirmed in Western blot analysis by determining cleavage of Bid and caspase-3 (Figure 7B). Cleavage of Bid as well as of caspase- 3 induced by incubation with $\mathrm{CyB}$ alone was inhibited in cells expressing the FADD-DN protein (Co, lane 4) compared to control cells transfected with the empty vector (Co, lane 2). CyB enhanced cleavage of Bid or caspase-3 induced either by UV radiation or anti-CD95 antibody treatment was found reduced upon transfection with FADDDN (UV, lane 4; CD95-Ab, lane 4) in comparison to mock transfected cells (UV, lane 2; CD95-Ab, lane 2).

\section{The effect of CyB is reduced in cells lacking CD95}

To corroborate the essential role of CD95 in CyB-induced apoptosis, epidermal cells from mice lacking CD95 (lpr-C3H) were used. $^{33}$ Compared to epidermal cells from wild-type mice, cells from Ipr mice were much less susceptible to the induction of apoptosis by СyВ (Figure 8). In addition, the enhancing effect of CyB on apoptosis induced either by UV radiation or by recombinant murine CD95L was remarkably suppressed in cells lacking CD95. 


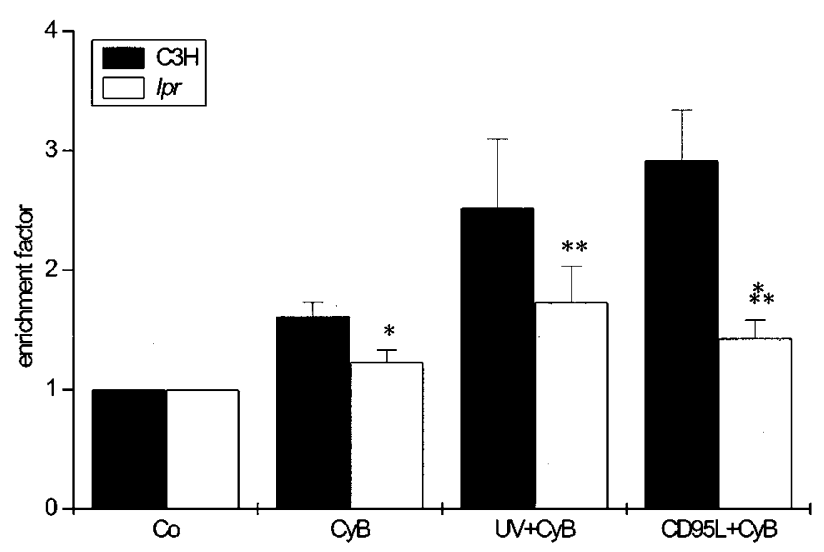

Figure 8 CD95 deficient cells are less susceptible to the effects of CyB. Epidermal cells were prepared from CD95 deficient (Ipr) or wild-type mice $(\mathrm{C} 3 \mathrm{H})$. Cells were left unstimulated or prestimulated with CyB. After $1 \mathrm{~h}$, cells were either left untreated for control purposes or treated with either $400 \mathrm{~J} / \mathrm{m}^{2}$ UV or with recombinant murine CD95 $(0.1 \mathrm{mg} / \mathrm{ml})$. Apoptotic cell death was evaluated $16 \mathrm{~h}$ later using a cell death detection ELISA. The enrichment factor was used as a parameter of apoptosis and is given as the mean \pm S.D. of three independently performed experiments. ${ }^{*} P<0.01$ vs $\mathrm{C} 3 \mathrm{H}(\mathrm{CyB})$, n.s. vs $\mathrm{Ipr}$ (Co); ${ }^{* *} P<0.01$ vs $\mathrm{C} 3 \mathrm{H}(\mathrm{UV}+\mathrm{CyB}) ;{ }^{* * *} P<0.001$ vs $\mathrm{C} 3 \mathrm{H}$ (CD95L+CyB)

These findings strongly support the hypothesis that disruption of the actin cytoskeleton by CyB causes random death receptor clustering, thereby triggering apoptosis. Enhancement of UV- and anti-CD95 antibody/CD95Ltriggered apoptosis by CyB results from intensified death receptor clustering following the same mechanism. Death receptor clustering induced or augmented by CyB appears to be functionally relevant, since disruption of the death receptor signaling pathway is associated with a loss of the apoptosis inducing/enhancing effect of CyB.

\section{Discussion}

Activation of the CD95 receptor either by UV radiation or by the cognate ligand, CD95L, or by agonistic antibodies is associated with receptor clustering. ${ }^{13,14}$ Receptor clustering is functionally relevant since inhibition coincides with reduced apoptosis. ${ }^{8,13,16}$ The underlying mechanism, however, still remains to be determined.

There is increasing evidence that receptor clustering is dependent on an interaction between the plasma membrane and the cytoskeleton. ${ }^{17}$ Changes in the flexibility of cell surface structure may be involved in the phenomenon of receptor clustering. Since actin is a cytoskeletal protein which is in the continual process of rapid polymerization and depolymerization in order to allow the morphological plasticity of living cells, ${ }^{22}$ we were interested in what impact the disruption of the actin cytoskeleton might have on UVinduced apoptosis, and whether death receptor clustering is affected. To disrupt the actin cytoskeleton, we used cytochalasins, fungal metabolites which block actin polymerization and thereby disturb existing actin filaments. ${ }^{34}$ The experiments presented in this paper were performed with cytochalasin $B(C y B)$, although similar observations were obtained when using cytochalasin $D$ (data not shown). In contrast, treatment of cells with phalloidin which stabilizes polymerized actin filaments did not affect apoptosis (data not shown).

Since treatment of particular cells with cytochalasins by itself induces apoptosis ${ }^{25-27}$ we utilized a concentration of CyB in the lower range. In fact, application of $50 \mu \mathrm{g} / \mathrm{ml}$ CyB induced only moderate apoptosis in HeLa cells. Suria et al. observed that cytoskeletal disruption by cytochalasin causes apoptosis by mechanism that requires ICE-like and caspase3 protease activities. ${ }^{26}$ Thus, the authors proposed that cytoskeletal disruption may cause the release and activation of caspase effector molecules or their activators from a storage compartment which ultimately leads to apoptotic cell death. In the present study, CyB-induced apoptosis was blockable by adding the caspase- 8 inhibitor zIETD. Since caspase- 8 is the enzyme farthest upstream in the CD95 signaling pathway and is also the crucial protease involved in CD95 signaling, ${ }^{35}$ this indicates that CyB might induce apoptosis in HeLa cells via activation of CD95.

Alternatively, CyB could cleave and thereby activate caspase-8 directly and independently of CD95. This appears unlikely since interruption of CD95 signaling by overexpressing a FADD-DN mutant was associated with a failure of CyB to induce apoptosis, suggesting that CyB causes apoptosis indeed via activation of CD95. These findings were further strengthened by the observation that the effect of CyB was suppressed in epidermal cells obtained from Ipr mice which lack CD95. If CyB would activate alternative pathways than CD95 clustering CyB would also have enhanced apoptosis in cells from CD95 lacking Ipr mice. Accordingly, confocal laser scanning microscopy revealed clustering of the CD95 receptor upon exposure to CyB. In this setting, it is unlikely that CD95 is activated by autocrine release of CD95L induced by $\mathrm{CyB}$, since addition of an antibody neutralizing CD95L did not inhibit CyB-mediated apoptosis (data not shown). Moreover, the clustering phenomenon is not due to increased surface expression of CD95 induced by CyB as determined by FACS analysis (data not shown). Therefore, we conclude that disruption of the actin cytoskeleton by СyB activates the CD95 receptor and thereby induces apoptosis. This demonstrates for the first time that CD95 cannot only be triggered by external stimuli but also by intracellular alterations, like disruption of the cytoskeleton.

Furthermore, exposure of HeLa cells to CyB for $1 \mathrm{~h}$ before UV irradiation resulted in an increased death rate. Since UVinduced apoptosis is driven by two major pathways, DNA damage and death receptor activation, we were interested in clarifying with which of the two pathways CyB might interfere. Accelerated removal of UV-mediated DNA lesions by inducing DNA repair via addition of the repair enzyme photolyase on the one hand caused reduction of cell death, as reported previously, ${ }^{8,11}$ but on the other hand did not alter the enhancing effect of CyB. This indicates that CyB enhances UV-mediated apoptosis independently of DNA damage. This is also supported by the observation that cell death caused by cisplatin which induces apoptosis preferentially by inducing DNA damage and not by affecting death receptors ${ }^{8,36}$ was not enhanced by CyB (data not shown).

The second major pathway utilized by UV radiation to induce apoptosis besides causing DNA damage is activation of membrane bound cell death receptors such as 
CD95. ${ }^{8,13,14}$ Activation of CD95 by either UV radiation or by an agonistic anti-CD95 antibody results in recruitment of the adaptor protein FADD followed by activation of the most upstream located caspase-8. Consequently, caspase-8 leads to cleavage of the downstream caspase- 3 on the one hand and to cleavage of Bid, which causes mitochondrial cytochrome $c$ release, on the other hand. ${ }^{30}$ Both of these membrane triggered pathways could be activated by CyB alone and were significantly enhanced after addition of CyB to either UV irradiation or application of the anti-CD95 antibody. Furthermore, the enhancing effect of CyB on UVor CD95-induced apoptosis was completely lost when CD95 signaling was interrupted either by addition of the caspase-8 inhibitor zIETD or by transfection of cells with a FADD-DN mutant. CyB alone or in combination with either UV irradiation or stimulation with CD95L was not able to induce or enhance apoptosis in epidermal mouse cells lacking the CD95 receptor (Ipr), supporting the concept that CyB exerts its apoptotic effect through random clustering of cell surface death receptors.

UV radiation also induces clustering of the tumor necrosis factor receptor- $1^{36}$ and of the TRAIL receptors 1 and 2 (own unpublished observations). Therefore, it is highly likely that CyB does not only activate CD95 but also tumor necrosis factor receptor-1 and the TRAIL receptors. This may also be the reason why keratinocytes from $I p r$ mice were strongly but not completely protected from the effect of CyB. Consequently, the findings presented in this study may not be confined to CD95 but may also apply for other death receptors.

Accordingly, increased clustering of the CD95 receptor following combined treatment with UV radiation and CyB was observed with confocal laser scanning microscopy. Overlay analysis localized CyB-induced CD95 clusters close to the same spots where maximum actin aggregation was observed. The fact that aggregation of nonfilamentous actin after disruption by CyB coincides with clustering of the cell surface death receptor CD95 suggests a link between CD95 and the actin cytoskeleton and that death receptor clustering may be related to cytoskeletal rearrangement. This is also supported by the observation that cells exposed to UV radiation did not only reveal CD95 receptor clustering but also slight actin aggregation (Figure 6). This phenomenon became even more obvious after incubation of cells with the agonistic anti-CD95 antibody. The molecular mechanism by which disruption of the cytoskeleton favors death receptor clustering still remains unclear.

Parlato et al recently demonstrated a linkage of CD95 to the actin cytoskeleton in human $\mathrm{T}$ lymphocytes. ${ }^{17} \mathrm{CD} 95$ susceptibility was associated with a constitutive polarized CD95 expression through an ezrin mediated association with the actin cytoskeleton. Treatment of T lymphocytes with ezrin anti-sense oligonucleotides or disruption of the cytoskeleton by cytochalasin D prevented CD95-mediated apoptosis. UVmediated cell death was not affected by treatment with cytochalasin $D$, thus being in contrast to our findings. However, it is important to note that cytochalasin D was used in a concentration $(0.5 \mu \mathrm{g} / \mathrm{ml})$ which by itself causes morphologic alterations but not apoptosis. In addition, Parlato et al $^{17}$ used lymphocytes, a cell type with strong migratory abilities with an increased turnover of actin filaments, while our studies were performed with HeLa cells which are less migratory. We selected an epithelial cell line for our studies, since UV-induced apoptosis is a phenomenon which in vivo almost exclusively occurs in the epidermis and thus is generally more relevant for epithelial cells.

Detachment from the extracellular matrix components may trigger apoptotic cell death as demonstrated for epithelial and endothelial cells. ${ }^{37,38}$ This type of apoptosis has been renamed anoikis. ${ }^{39}$ Anoikis results from activation of the CD95 pathway by upregulation of CD95L and by downregulation of FLIP. ${ }^{37}$ Although disruption of the actin cytoskeleton may induce detachment from extracellular components, differences appear to exist since treatment of HeLa cells with CyB was neither associated with an upregulation of CD95L nor with a downmodulation of FLIP.

Taken together, the present findings demonstrate that disruption of the cytoskeleton in epithelial cells causes apoptosis via activation of the death receptor CD95, indicating that death receptors can also be triggered from inside of the cell. External trimerisation of CD95 by either an anti-CD95 antibody or UV radiation results in moderate intracellular rearrangement of cytoskeletal actin filaments, indicating a functional connection between cell death receptor clustering and actin aggregation in the cell periphery under normal apoptotic conditions. Furthermore, induction of an intracellular cytoskeletal disruption by CyB also results in cell peripheral aggregation of depolimerized actin coinciding with cell death receptor clustering in the absence of an external apoptotic stimulus. Accordingly, combination of either one of the external stimuli, i.e. either UV radiation or anti-CD95 antibody, with intracellular rearrangement of actin cytoskeleton induced by CyB causes death receptor clustering in an additive way, thereby enhancing apoptosis. Thus, it may be speculated that rearrangement of the cytoskeletal actin filaments forms the basis for cell death receptor clustering.

UV-induced apoptosis is regarded as a protective phenomenon to eliminate DNA-damaged cells which are predisposed to malignant transformation. ${ }^{40}$ Thus, dysregulation of UV-mediated cell death would be expected to confer an increased risk of UV-induced skin cancer. Greater understanding of the underlying control mechanisms will allow clarification of the complex pathways which are intertwined in the generation of apoptotic cells following solar/UV exposure. By demonstrating that alterations of the cytoskeleton trigger death receptors and that this may influence UV-induced apoptosis, the present study identifies an additional pathway involved in the complex process of UV-induced apoptosis.

\section{Materials and Methods}

\section{Cells and reagents}

The human epithelial carcinoma cell line HeLa (American Tissue Culture Collection, Rockville, MD, USA) was cultured in RPMI 1640 with $10 \%$ FCS. Before stimulation cells were kept in serum free medium for $24 \mathrm{~h}$. Cells were exposed to UV radiation through 
colourless medium without FCS. For UV irradiation a bank of six TL12 fluorescent bulbs (Philips, Eindhoven, The Netherlands) was used which emit most of their energy within the UVB range $(290-320 \mathrm{~nm})$ with an emission peak at $313 \mathrm{~nm}$. Throughout this study a dose of $400 \mathrm{~J} / \mathrm{m}^{2}$ was used. Control cells were subjected to the identical procedure without being exposed to UV radiation.

Epidermal cells from ears of CD95 deficient $(/ p r-\mathrm{C} 3 \mathrm{H} / \mathrm{HeN})^{33}$ or wild-type mice $(\mathrm{C} 3 \mathrm{H} / \mathrm{HeN})$ were prepared as described previously. ${ }^{41}$ Briefly, ears were separated, incubated in PBS containing $0.25 \%$ trypsine at $37^{\circ} \mathrm{C}$ for $1.5 \mathrm{~h}$ and single cell suspensions were obtained.

Disruption of filamentous actin of the cytoskeleton was induced with $50 \mu \mathrm{g} / \mathrm{ml}$ cytochalasin B (Sigma Corp., St. Louis, MO, USA). Activation of caspases was blocked using the specific oligopeptides (20 $\mu \mathrm{M}$ each) $\mathrm{z}$-Val-Ala-Asp- $\mathrm{CH}_{2}$ (z-VAD) for ICE proteases, z-lle-GluThr-Asp- $\mathrm{CH}_{2} \mathrm{~F}$ (z-IETD) for caspase-8, or z-Leu-Glu-His-Asp- $\mathrm{CH}_{2} \mathrm{~F}$ (zLEHD) for caspase-9 (all Enzyme Systems Products, Livermore, CA, USA). To induce CD95-mediated apoptosis, an agonistic mouse IgM antibody directed against human $\mathrm{CD} 95(\mathrm{CH}-11$, Immunotech, Luminy, France) was added to the cell medium at a final concentration of $1 \mu \mathrm{g} /$ $\mathrm{ml}$. Recombinant murine CD95L was obtained from Alexis (San Diego, CA, USA). The plasmid allowing overexpression of a FADD dominant negative mutant (pcDNA3-FADD-DN) was kindly provided by $V$ Dixit (Genentech, San Francisco, CA, USA).

\section{Induction of DNA repair via photoreactivation}

Exogenous induction of DNA repair via photoreactivation was performed as described previously in detail. ${ }^{8}$ Briefly, photolyase was encapsulated into liposomes (Photosomes ${ }^{\circledR}$, AGI Dermatics, Freeport, NY, USA) at a concentration of $1.2 \mathrm{mg} / \mathrm{ml} .{ }^{42}$ Liposomes consisted of the lipids egg phosphatidylcholine, egg phosphatidyl trans-ethanolamine, oleic acid and the membrane stabilizer cholesterol hemisuccinate. Empty liposomes were used as negative controls, referred to as liposomes. For photoreactivation, HeLa cells were irradiated as described above and either photosomes or liposomes $\left(40 \mu \mathrm{l} / \mathrm{ml}\right.$ each) were added. Cells were incubated at $37^{\circ} \mathrm{C}$ for $1 \mathrm{~h}$ in the dark followed by illumination with photoreactivating light. As a light source for photoreactivating light, UVA fluorescent bulbs (TL09, Philips) filtered through a $6 \mathrm{~mm}$ glass plate with peak emission at $365 \mathrm{~nm}$ were used. Cells were exposed for $20 \mathrm{~min}$ which corresponds to a photoreactivating light fluence of $12 \mathrm{~kJ} / \mathrm{m}^{2}$. After photoreactivation, cells were supplemented with normal RPMI containing 10\% FCS and incubated for $16 \mathrm{~h}$ at $37^{\circ} \mathrm{C}$.

For Southwestern dot-blot analysis, genomic DNA was extracted from cells according to the protocol from Biozym Diagnostic (Hessisch Oldendorf, Germany) $1 \mathrm{~h}$ after stimulation and DNA damage was detected utilizing an antibody directed against thymine dimers (Kamiya Biomedical, Thousand Oaks, CA, USA) as described previously. ${ }^{8}$

\section{Detection of cell death}

Sixteen hours after stimulation cells were detached from dishes, and apoptosis analyzed by a cell death detection ELISA (Boehringer Mannheim, Mannheim, Germany). The enrichment of mono- and oligonucleosomes released into the cytoplasm of cell lysates is detected by biotinylated anti-histone- and peroxidase-coupled antiDNA-antibodies and is calculated as follows: absorbance of sample cells/absorbance of control cells. Unless otherwise stated, this factor was used as a parameter of apoptosis and is given as the mean \pm S.D. of three independently performed experiments. An enrichment factor of 1.0 corresponded approximately to $5-7 \%$ of dead cells as determined in parallel by FACS analysis after staining with FITC labeled annexin V (Bender Corp., Vienna, Austria).

\section{Western blot analysis}

For total protein extracts, cells were lysed in lysis-buffer $(50 \mathrm{mM}$ HEPES pH 7.5, $150 \mathrm{mM} \mathrm{NaCl}, 10 \%$ glycerol, 1\% Triton-X-100, $1.5 \mathrm{mM}$ $\mathrm{MgCl}_{2}, 1 \mathrm{mM}$ EGTA, $100 \mathrm{mM} \mathrm{NaF}, 10 \mathrm{mM}$ pyrophosphate, $0.01 \%$ $\mathrm{NaN}_{3}$ and complete ${ }^{\mathrm{TM}}$ protease inhibitor cocktail) and were subjected to sonication. Specific cytoplasmic protein extracts revealing mitochondrial cytochrome $c$ release into the cytoplasm were obtained by lysing cells in buffer consisting of $250 \mathrm{mM}$ sucrose, $80 \mathrm{mM} \mathrm{KCl}$ and $1 \mu \mathrm{g} / \mathrm{ml}$ digitonin (Sigma) and passing them through a 26 gauge needle eight times. After centrifugation, supernatants were collected, and the protein content measured by BioRad Protein assay kit (BioRad, Hercules, CA, USA). Mitochondrial proteins were extracted utilizing a mitochondrial fractionation kit (Alexis). Thirty $\mu \mathrm{g}$ of the respective cytosolic and $50 \mu \mathrm{g}$ of the mitochondrial protein samples were subjected to either 12 or $13 \%$ SDS-PAGE, blotted onto nitrocellulose membranes and incubated with antibodies directed against caspase-3 (Dianova, Hamburg, Germany), Bid (R\&D Systems Inc., Minneapolis, MN, USA), cytochrome c (Biosource, Nivelles, Belgium) or FLIP (Pharmingen, San Diego, CA, USA). Equal loading was determined by reprobing membranes with an antibody directed against $\alpha$-tubulin (Calbiochem, San Diego, CA, USA). Signals were detected with an $\mathrm{ECL}^{\mathrm{TM}}$-kit (Amersham, Buckinghamshire, UK).

\section{Transfection}

HeLa cells $\left(1 \times 10^{7}\right)$ were washed once with PBS and resuspended in $1 \mathrm{ml}$ RPMI medium/2.5\% DMSO. Cells were electroporated with $25 \mu \mathrm{g}$ of each plasmid DNA pcDNA3-FADD-DN or the empty vector pcDNA3CMV according to the method described by Melkonyan et $a .^{43}$ Transfection efficacy of cells cotransfected with a plasmid encoding $\beta$ galactosidase (pCMV- $\beta$ gal; Stratagene, La Jolla, CA, USA) was determined $24 \mathrm{~h}$ later by staining cells with X-gal $(100 \mu \mathrm{g} / \mathrm{ml})$ in $5 \mathrm{mM}$ potassium ferricyanide, $5 \mathrm{mM}$ potassium ferrocyanide, and $1 \mathrm{mM}$ $\mathrm{MgCl}_{2}$ in PBS. Transfection efficiency ranged from 30 to $50 \%$.

\section{Confocal laser scanning microscopy}

Cells were seeded into tissue culture chambers (Chamber Slide; Nunc, Naperville, IL, USA). After culturing without FCS for $24 \mathrm{~h}$, cells were preincubated with CyB for $1 \mathrm{~h}$ followed by either UV irradiation or stimulation with an agonistic antibody against CD95. Control cells were left untreated. Two hours after stimulation impermeated cells were fixed for 30 min in 3.5\% formaldehyde in PBS, pH 7.4, incubated for $5 \mathrm{~min}$ in $0.1 \mathrm{M}$ glycine and blocked with $1 \% \mathrm{BSA} / 50 \% \mathrm{NGS}$ (Dianova, Hamburg, Germany) for $1 \mathrm{~h}$ at room temperature. Subsequently cells were incubated with an antibody directed against CD95 (ZB4, Upstate Biotechnology Inc., Lake Placid, NY, USA) 1:20 in $1 \% \mathrm{BSA}$ for $1 \mathrm{~h}$ at room temperature, followed by washing in PBS and incubation with the secondary FITC-labeled goat anti-mouse IgG (DAKO Diagnostica GmbH, Hamburg, Germany) 1:10 in 1\% BSA for $45 \mathrm{~min}$ at room temperature. Thereafter, intracellular actin staining was performed. For this purpose, TRITC-conjugated phalloidin was added for $30 \mathrm{~min}$ at room temperature. Phalloidin was dissolved in $1 \%$ DMSO at a concentration of $1 \mu \mathrm{g} / \mathrm{ml}$ to enable penetration into impermeated cells. The specimens were analysed with a confocal laser scanning microscope equipped with an argon-krypton laser (Leica TCS Confocal Systems) and a $63 \times$ water immersion objective (detailed description of laser scanning microscopy in ref. ${ }^{44}$ ). FITC was excited with the $488 \mathrm{~nm}$ line and TRITC with the $568 \mathrm{~nm}$ line and the preconfigurated filter setup from Leica for FITC and TRITC were used. The cells were $x / y$ scanned in the fluorescence mode with $10 \mathrm{~mW}$ laserpower for $2 \mathrm{~s}$. The pinhole was adjusted to collect the fluorescence of an optical section with an equal thickness for all 
images less than $0.8 \mu \mathrm{m}$. Four $512 \times 512$ pixel images were averaged to obtain the FITC/TRITC overlays. FITC and TRITC fluorescence intensities are displayed separately in glow overflow pseudocolor images according to the respective intensity scale bars indicated on top (see Figure 6).

\section{Acknowledgements}

We are grateful to Drs A Mehling and E Zeise for helpful discussions, Dr D Yarosh for providing Photosomes ${ }^{\mathbb{R}}$, Dr V Dixit for providing the FADD-DN construct and Drs Vestweber and Klämbt for providing confocal laser scanning microscopy facilities. This work was supported by grants from the Federal Ministry of Education and Research (07UVB63A/5) and the European Community (ENV4CT97-0556).

\section{References}

1. Ashkenazi A and Dixit VM (1998) Death receptors: signalling and modulation. Science 281: 1305-1308

2. Boldin MP, Varfolomeev EE, Pancer Z, Mett IL, Camonis JH and Wallach D (1995) A novel protein that interacts with the death domain of Fas/APO1 contains a sequence motif related to the death domain. J. Biol. Chem. 270: 7796-7798

3. Chinnaiyan AM, O'Rourke KO, Tewari M and Dixit VM (1995) FADD, a novel death domain-containing protein, interacts with death domain of Fas and initiates apoptosis. Cell 81: 505-512

4. Boldin MP, Doncharwov TM, Gotsev YV and Wallach A (1996) Involvement of $\mathrm{MACH}$, a novel MORTI/FADD-interactin protease, in Fas/APO/1- and TNF receptor-induced cell death. Cell 85: 803-815

5. Muzio M, Chinnaiyan AM, Kischkel FC, O'Rourke K, Shevchenko A, Ni J, Scaffidi C, Bretz JD, Zhang M, Gentz R, Mann M, Krammer PH, Peter ME and Dixit VM (1996) FLICE, a novel FADD-homologous ICE/CED-3 like protease, is a recruited to the CD95 (Fas/APO-1) death-inducing signaling complex. Cell 85: $817-827$

6. Salvesen GS and Dixit VM (1997) Caspases: Intracellular signaling by proteolysis. Cell 91: $443-446$

7. Kulms D and SchwarzT (2000) Molecularmechanisms of UV-induced apoptosis. Photodermatol. Photoimmunol. Photomed. 16: 195-201

8. Kulms D, Pöppelman B, Yarosh D, Luger TA, Krutmann J and Schwarz T (1999) Nuclear and cell membrane effects contribute independently to the induction of apoptosis in human cells exposed to UVB radiation. Proc. Natl. Acad. Sci. USA 96: $7974-7979$

9. Ley RD and Applegate LA (1985) Ultraviolet radiation-induced histopathologic changes in the skin of the marsupial Monodelphis domestica. II. Quantitative studies of the photoreactivation of induced hyperplasia and sunburn cell formation. J. Invest. Dermatol. 85: 365-367

10. Schwarz A, Ständer S, Berneburg M, Böhm M, Kulms D, van Steeg H, GroßeHeitmeyer K, Krutman J and Schwarz T (2002) Interleukin-12 suppresses ultraviolet radiation-induced apoptosis by inducing DNA repair. Nat. Cell. Biol. 4: 26-31

11. Stege H, Roza L, VinkAA, Grewe M, Ruzicka T, Grether-Beck S and Krutmann J (2000) Enzyme plus light therapy to repair DNA damage in ultraviolet-Birradiated human skin. Proc. Natl. Acad. Sci. USA 97: 1790-1795

12. Ziegler A, Jonason JS, Leffel DW, Simon JA, Sharma HW, Kimmelman J, Remington L, Jacks T and Brash DE (1994) Sunburn and p53 in the onset of skin cancer. Nature 372: 773-776

13. Aragane $Y$, Kulms D, Metze D, Wilkes G, Pöppelmann B, Luger TA and Schwarz $T$ (1998) Ultraviolet light induces apoptosis via direct activation of CD95 (Fas/ APO-1) independently of its ligand CD95L. J. Cell. Biol. 140: 171-182

14. Rehemtulla A, Hamilton CA, Chinnaiyan AM and Dixit VM (1997) Ultraviolet radiation-induced apoptosis is mediated by activation of CD-95 (Fas/APO-1). J. Biol. Chem. 272: 25738-25786

15. Rosette C and Karin M (1996) Ultraviolet light and osmotic stress: activation of the JNK cascade through multiple growth factor and cytokine receptors. Science 274: $1194-1197$
16. Kulms D, Pöppelmann B and Schwarz T (2000) Ultraviolet radiation-induced interleukin 6 release in HeLa cells is mediated via membrane events in a DNA damage independent way. J. Biol. Chem. 275: 15060-15066

17. Parlato S, Giammarioli AM, Logozzi M, Lozupone F, Matarrese $P$, Lucani $F$, Falchi M, Malorni W and Fais S (2000) CD95 (APO-1/Fas) linkage to the actin cytoskeleton through ezrin in human $T$ lymphocytes: a novel regulatory mechanism of the CD95 apoptosis pathway. EMBO J. 19: 5123-5134

18. Drubin DG and Nelson WJ (1996) Origins of cell polarity. Cell 84: $335-344$

19. Kusumi A and Sako $Y$ (1996) Cell surface organization by the membrane skeleton. Curr. Opin. Cell. Biol. 8: 566-576

20. Luna EJ and Hitt AL (1992) Cytoskeleton-plasma membrane interactions. Science 258: $955-963$

21. Tsukita A, OishiK, Sato N, Sagara J, Kawakai A and Tsukita S (1994)ERM family members as molecular linkers between the cell surface glycoprotein CD44 and actin-based cytoskeletons. J. Cell. Biol. 126: 391-401

22. Maruyama W, Irie S and Sato TA (2000) Morphological changes in the nucleus and actin cytoskeleton in the process of Fas-induced apoptosis in Jurkat T cells. Histochem. J. 32: 495-503

23. Mashima T, Naito M and Tsuruo T (1999) Caspase-mediated cleavage of cytoskeletal actin plays a positive role in the process of morphological apoptosis. Oncogene 18: 2423-2430

24. Stegh AH, Herrmann H, Lampel S, Weisenberger D, Andra K, Seper M, Wiche G, Krammer PH and Peter ME (2000) Identification of the cytolinker plectin as a major early in vivo substrate for caspase 8 during CD95- and tumor necrosis factor receptor-mediated apoptosis. Mol. Cell. Biol. 20: 5665-5679

25. Rao JY, Jin YS, Zheng Q, Cheng J, Tai J and Hemstreet III GP (1999) Alterations of the actin polymerisation status as an apoptotic morphological effector in $\mathrm{HL}-60$ cells. J. Cell. Biochem. 75: 686-697

26. Suria H, Chau LA, Negrou E, Kelvin DJ and Madrenas J (1999) Cytoskeletal disruption induces $T$ cell apoptosis by a caspase-3 mediated mechanism. Life Sci. 65: 2697-2707

27. YamazakiY, Tsuruga M, Zhou D, Fujita Y, Shang X, Dang Y, KawasakiKand Oka $S$ (2000) Cytoskeletal disruption accelerates caspase-3 activation and alters the intracellular membrane reorganization in DNA damage-induced apoptosis. Exp. Cell. Res. 259: 64-78

28. Yarosh D, Bucana C, Cox C, Alas L, Kibitel J and Kripke ML (1994) Localization of liposomes containing a DNA repair enzyme in murine skin. J. Invest. Dermatol. 103: $461-468$

29. Eker AP, Kooiman P, Hessels JK and Yasui A (1990) DNA photoreactivation enzyme from the cyanobacterium anacystis nidulans. J. Biol. Chem. 15: 80098015

30. Los M, Wesselborg S and Schulze-Osthoff K (1999) The role of caspases in development, immunity, and apoptosis signal transduction: Lessons from knockout mice. Immunity 10: 629-639

31. Irmler M, Thome M, Hahne M, Schneider P, Hofmann K, Steiner V, Bodmer JL, Schroter M, Burns K, Mattmann C, Rimoldi D, French LE and Tschopp J (1997) Inhibition of death receptor signals by cellular FLIP. Nature 388: 190-195

32. Scaffidi C, Schmitz I, Krammer PH and Peter ME (1999) The role of c-FLIP in modulation of CD95-induced apoptosis. J. Biol. Chem. 274: 1541-1548

33. Watanabe-Fukunaga R, Brannan $\mathrm{Cl}$, Copeland NG, Jenkins NA and Nagata S (1992) Lymphoproliferation disorder in mice explained by defects in Fas antigen that mediates apoptosis. Nature 356: 314-317

34. Walling EA, Krafft GA and Ware BR (1988) Actin assembly activity of cytochalasins and cytochalasin analogs assayed using fluorescence photobleaching recovery. Arch. Biochem. Biophys. 264: 321-332

35. Peter ME and Krammer PH (1998) Mechanisms of CD95 (APO1/Fas)-mediated apoptosis. Curr. Opin. Immunol. 10: 545-551

36. Sheikh MS, Antinore MJ, Huang Y and Fornace Jr AJ (1998) Ultravioletirradiation-induced apoptosis is mediated via ligand independent activation of tumor necrosis factor receptor 1. Oncogene 17: 2555-2563

37. Aoudjit F and VuoriK (2001) Matrix attachment regulates Fas-induced apoptosis in endothelial cells: a role for c-flip and implications for anoikis. J. Cell. Biol. 152 $633-643$

38. Rosen K, RakJ, Leung T, Dean NM, KerbelRS and Filmus J (2000)Activated Ras prevents downregulation of $\mathrm{Bcl}-\mathrm{X}(\mathrm{L})$ triggered by detachment from the extracellular matrix. A mechanism of Ras-induced resistance to anoikis in intestinal epithelial cells. J. Cell. Biol. 149: 447-456

39. Frisch SM and Francis H (1994) Disruption of epithelial cell-matrix interactions induces apoptosis. J. Cell. Biol. 124: 619-626 
40. Kraemer KH (1997) Sunlight and skin cancer: another link revealed. Proc. Natl. Acad. Sci. USA 94: 1-14

41. Stingl LA, Sauder DN, lijima M, Wolff K, Pehamberger H and Stingl G (1983) Mechanism of UV-B-induced impairment of the antigen-presenting capacity of murine epidermal cells. J. Immunol. 130: 1586-1591

42. Ceccoli J, Rosales N, Tsimis J and Yarosh DB (1989) Encapsulation of the UVDNA repair enzyme $T 4$ endonuclease $V$ in liposomes and delivery to human cells. J. Invest. Dermatol. 93: 190-194
43. Melkonyan H, Sorg C and Klempt M (1996) Electroporation efficiency in mammalian cells is increased by dimethyl sulfoxide (DMSO). Nucl. Acids Res. 24: $4356-4357$

44. Pawley JB (1990) Handbook of biological confocal microscopy (New York: Plenum Press). 